\title{
Die Frau im öffentlichen Leben
}

Anna Pappritz

Follow this and additional works at: https://scholarsarchive.byu.edu/sophnf_essay

Part of the German Literature Commons

\section{BYU ScholarsArchive Citation}

Pappritz, Anna, "Die Frau im öffentlichen Leben" (1909). Essays. 1731.

https://scholarsarchive.byu.edu/sophnf_essay/1731

This Article is brought to you for free and open access by the Nonfiction at BYU ScholarsArchive. It has been accepted for inclusion in Essays by an authorized administrator of BYU ScholarsArchive. For more information, please contact scholarsarchive@byu.edu, ellen_amatangelo@byu.edu. 


$$
f_{c} \frac{432}{20}
$$




\section{Dolitische Bildung fiir alle Rreise der Bevolkerung!}

Cejt und verbreitet die Vollss. ichiniften des. nationalvereins fur bas liberale Deut obland:

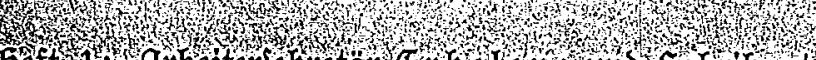

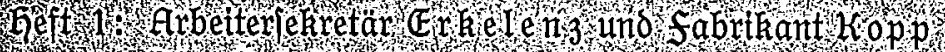
Die al beiterfrage, 10 . p.g.

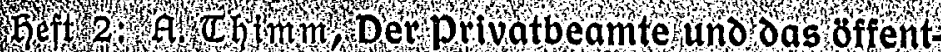
iche reben, $10 \%$ ofg:

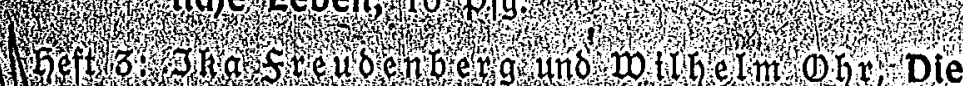

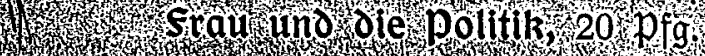

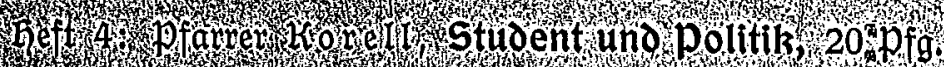

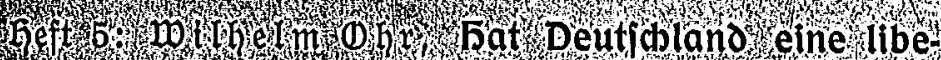
rale 3ukunt? 20 i of 9 ?

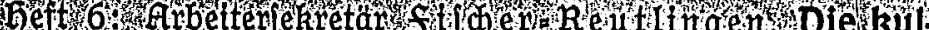

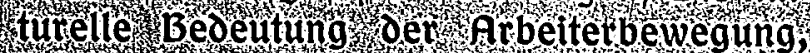

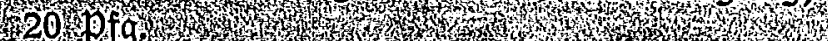

in allen Buchbanolingen oder ourd den vierláa

Burou: Tationaloerein Micine en, finenitrabe 3 .

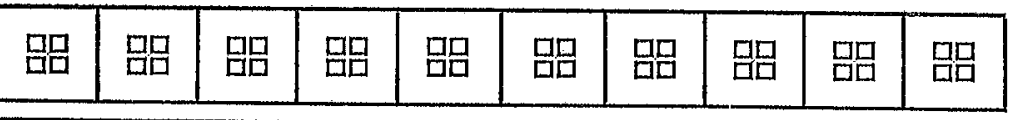

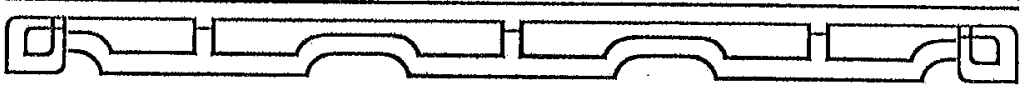

\section{Whong

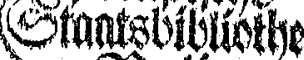 20ettin \\ Die frau im öffentlichen Seben.}

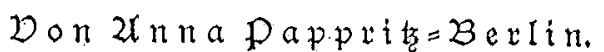

D

as Jahr 1907 hat uns frauen einen gropen fortfdrtt

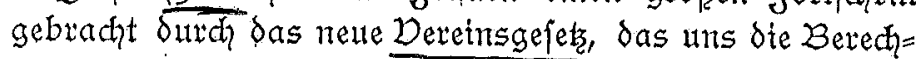
tigung zur Tethtahme ait politifthen Deretiten wnd Dex fammlungen gegeben hat. Wir frauen find dadurd politif a mündig geworden. Wir frenen uns diefes fortfartttes und wir danfen es den liberalen zlämmen, daf fte mit uns und füt uns biefes Recht erfämpft haben; aber wit betrad ten diefen foutfdrtt bod eben mut als den alleverften Shutt. Whir frauen beftrden uns nut in der Sage der Weltumfegles: alter Jetten, die zum erftenmal den fuf auf das fefte Sand der neuen welt fetzten: Das Land threr

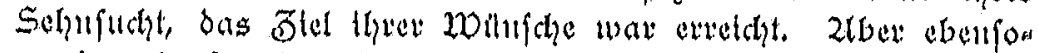
twenig wie filx jente die Erreidung diejes Jieles ein $\mathfrak{Z}_{\text {ustuhen }}$ bedentete, ebenfowentg danf dies für uns det fall fein. Jmt Gegentetl, die rechte 2lrbeit foll tent füt uns erft beginnen: Die frudtbarmadung des neugewonnenen Ge= bietes, Daju werbent uns die liberalen Wänner ftherlich thren Beiftand nidht verfagen, fondern fie werden uns helfen,

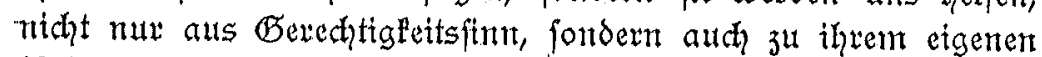
Bejten und in Intereffe des Dolpsganzen. Dent dev deutfine 
2Tamn will, indem ev den frauen das Recht der politifhen Der= eintgung gab, dodh nicht ledtglidh die Dereinsmeieret verbreitent

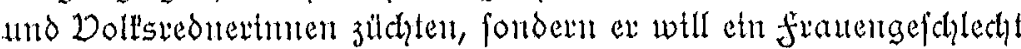
erziehen, das mit ihm int politifdhent $\mathfrak{S e b}_{\text {en }}$ witken und arbeiten

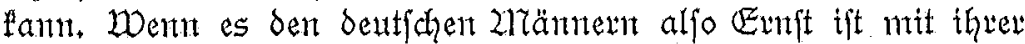
Itberalen Gefinnung unt die Betonung ifrer demofratifhen prin= zipien etwas anderes ift, als leeve Sdhönedneret, fo müffen fie aud folgerthtis fehr bald $\delta$ en zwetten Sdritt tun und ben framen $\delta$ as Redht geben, unt das wir fhont fo lange bitten,

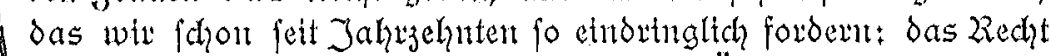

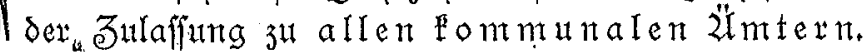

Zlls por Jahyesfrift der Zrattontalverein fult das liberale Deutfhland gegründet wurde, da bejeidntete fran 2riartanne

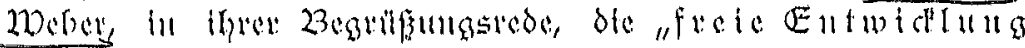

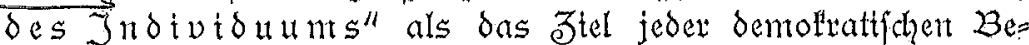
wegung und fie forderte, aus Gründen der Gerechtigleit, drefe

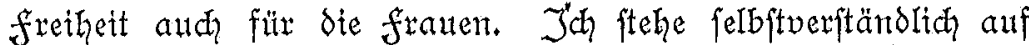
ganz dentfelben Standpunft, aber idh möhte die 2notivierung diefes Bedankents tod näher ansführen. Woin franen fordern Sas Recht auf fiete Eutfaltung nitht nux, unt Teil zu haben

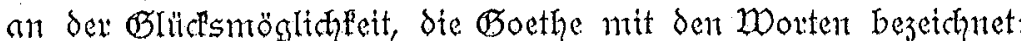

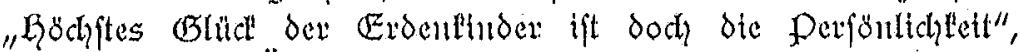

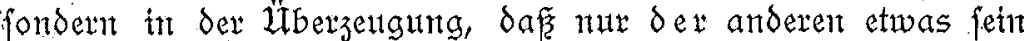
fanu, der felbft etwas geworden tit. 2tnd wir wollen andern efwas feit: wiv wollent thjere Zrbeit, unfere Kraft in den Dienjt tunferes Daterlandes ftellen. Das förnen wir aber nux, wenn

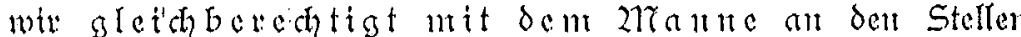

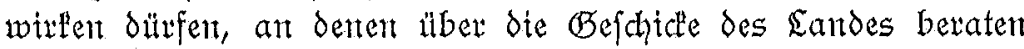
w1to entfotedent wito.

Whir haben als Joeal unferer freten Bewegung "ote frete

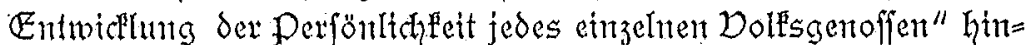
geftell, ich betone ausdrülich, jedes eituzeluen Dolfsgenofien, benn wir wollen ntcht nux an uns denfen, an die Dextreter des gebilloeten Bürgertums, fondent wir wollen aud fämptent für die freiheit und Wohlfahut der arbeitenden Klaffen. Kilaffenhaf und $\mathbb{E}_{\mathfrak{g}}$ oismus haben ehte thefe Kluft gegraben zwifhen unferm
Bürgertum und den arbeitenden Klaffent. Wiv wollen heute niaft

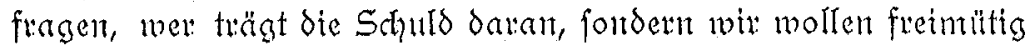

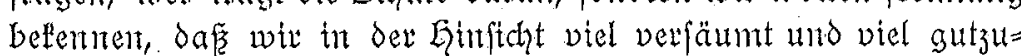
machen habent. Das 27ratertal aber, mit dent wir diefe Zuluft ausfillfen fömren, heifít Sozialrefornt in weiteften Sinne. Und gerade für dieje

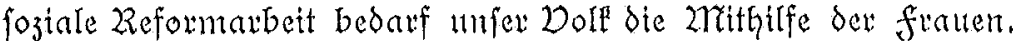

(Breifen Sie irgend eit Teilgebiet heraus aus ber gropzent fozialen frage, legen Sie Jhre Gand auf irgent ente ber vielen munden punte ant Lröper des Dolfes, mis Sie werden befemen mulfen: Gien tut frauengilfe not! Denfen Sie allein

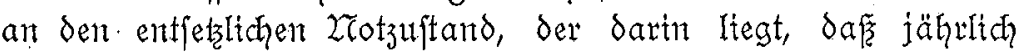
bet uns 450000 Kinder, aljo faft einte Galbe Wrifliton Kinder in Zlter bis zat wier Jahren fterbent Welche Dergendung an

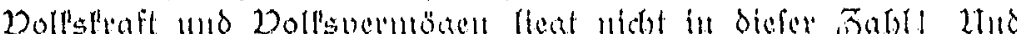

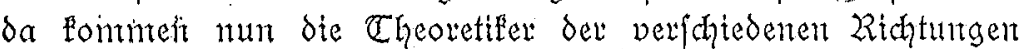
und doftorn an diefem 2rotzuftand berum. Die einen fagen: die Säuglingsfterbitchleit wädhft int Derhältnis zur Geburten= ziffer; wir müffen die Kinderzahl einfhränten; je wentger Zitndev geboren werden, je meht haben Chanten am Eeben zu bleibert. Die andern aber wettern gegen den Zeu=2ntalthuftantsmus und

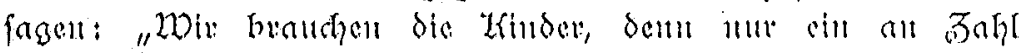
wachfendes Dolf ift ein an 21Tacht und Einflufis wachfendes Dolf." Foch. ftehen wohI die meifter framen auf diejent Standpunft,

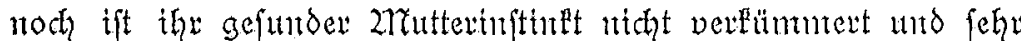
Untred habent die 2räntuter, went fie dent frauten den Dorwunf des Egoismus madhen und fagen, die frauen wolltent die zlühen

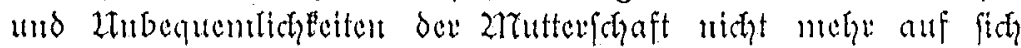
nehment. Der Zeu=27Talthuftantisntus ift nthet geboren worden

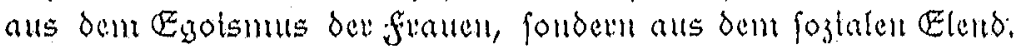

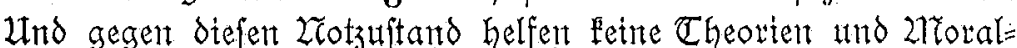
predigten, fondern mur prattifhe Reformen. Die 27ränter aber

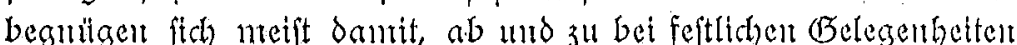

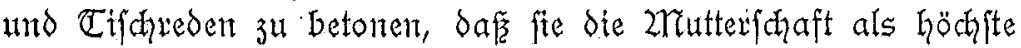
Seiftung ber frau hoch unt heiltg halten. Del widhtiger wäre es jedody, duwh die $\mathbb{C}$ at zu zeigen, dafiz fie diefe frauenleiftung

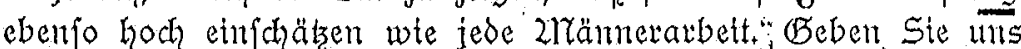




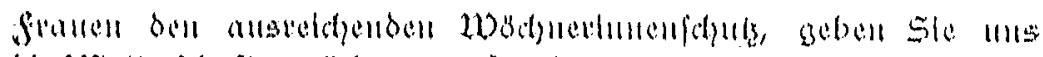

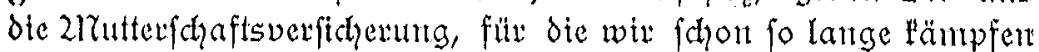
und Sie werden fehen, dafis die fual aud threrfetts den höaftent Stolz und ifre Befriedigutg datit finden wirb, dem Daterlande

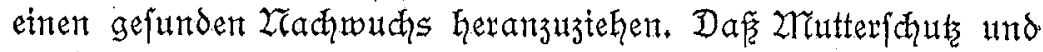

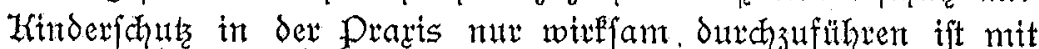

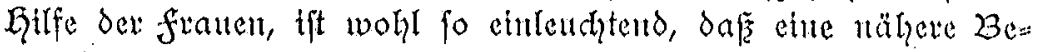

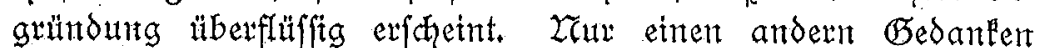

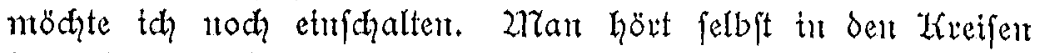
der líberalen 2ränner manchmal die Befürattung laut werdent, dafif die Bew Fönnte. Dieje Befürditung ift wohl unbegüindet, dent die natür= Itde Deranlagung bev frau wird ftets etue famtiltengrütoung

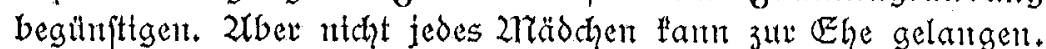
Daher milffen win benen, dte nidht heiraten fönten oder wollen

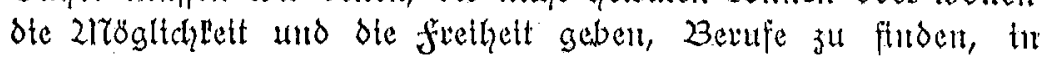

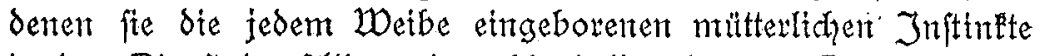

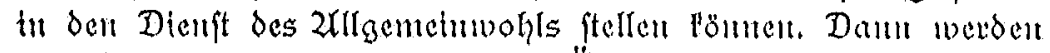

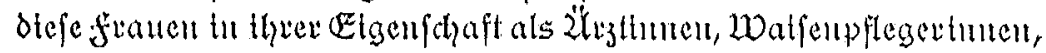

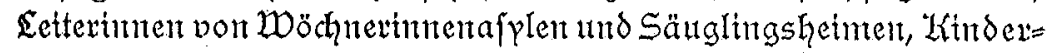
horten und Kindergärten wiw. taufende nnd abertanjende vont

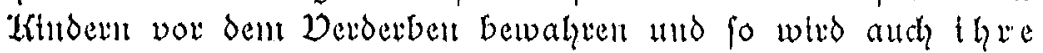
Zrbeit früdte tragen tm Sinne eitter Kraftfteigerung unferes Dolfes.

Dev zweite wunde punt, an bem frauentiffe not tut, ift Jer dev wadfenden Derwahrlofung und Kriminalität unferer Jugend. 50000 Kinder zwifhen 12 und 18 Jahren werdent jährlich verunteilt, und die Statiftił lehut uns, da jein grofier Tetl diefer exitmaltg Damuteilten zu Bewohnheitsverbrechew wird. Und doch find dieje Kinder in den meiften fällen nidht fhledht, unt verwahrloft, nangelhaft oder gar nidit erzogent. Ste wiltdent 3ut retten feint, went frauenhilfe jut rednten Jett einträte. Woht haben bie meiften Bundesftaaten hl lezter Zett Beferze gegeben zum Schuz dev Luinder: bas fürjorgeerziehungsgejez und das Gefeł зu: Befdräntung der gewerblthen Kinderarbett. Went

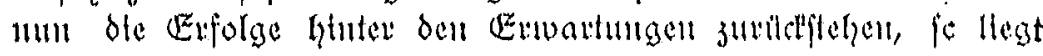

Das daran, bafiz es an geeigneten familien, 2lnjtalten und Pflegern

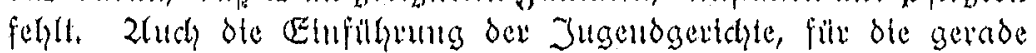
von fetten dex franen fo lebhaft Propaganda gemant worben ift, ftellt wiederum grope 2lnforderungen an die weibliche Setitungs= Araft und Opferwilligfeit, dent mit Recht betont Strafrechtslehrer Prof. v. Kiszt, dafif der Gauptfartor bet den Jugendgetiditshöfen die fürforgeausfaulfo unter 2hithilfe der fratten find. Worden bie frawen btefen anfordenungen gewadfen fetn? Das hängt nidit in erfter sinie pon ifnen felbit $a b$, fondern zunt grofzen

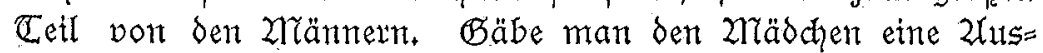

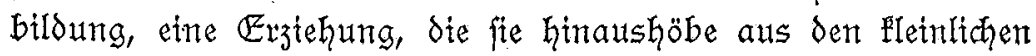
und fripolen Jnteveffenfphären det frath, gäbe nant uns die

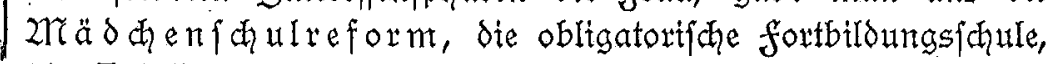

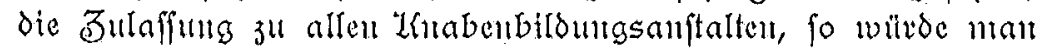
ein frauengefdilecht heranbilden, bas fid fetmer Pflithten gegen

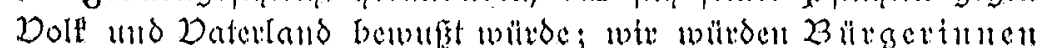
exztehert, dte aud in Gaushalt des Staates ihren Platz ausju= fillen vermögen. Diefe poften aber, für die wiv dte frauett=

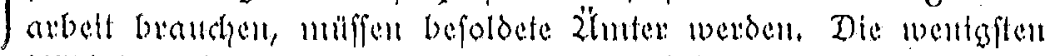
27ädchen fonmten als Rentterimen auf bte Welt und bet bem gröften Joealismus ift es ifnen unmöglich, ihre 2lubett umfonft in den Dienft des Dolfswohls zu ftellen; aidh fie milffen nadh

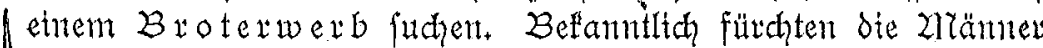
vielfad die Konluretr dev frau; num wohl, hier ift thrten ein Zrittel gegeben, fith diefe Konturenz der frauen zum grofien Teil vom Galfe zu fdaffen, inden fie ifnen bejoldete Zौnter gebent, in denert die frauen ihre fpezififin weibliche Begabung in den Dienft des Daterlandes ftellen Fömen, ans denen fie Peine Znännew verorängen und wo fie befferes, eigenartigeres leiften pömen, als dev 27antr.

Unto nun Bomme ich zum dritten Punft, wo meines $\mathbb{E}_{\mathfrak{r}}=$ adytens die frauentilfe not tut. Das ift die frage ber offent= frichen Sittlichlett. Was haben die Znänner aus ihl gentacht?

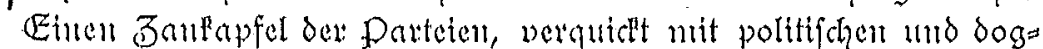

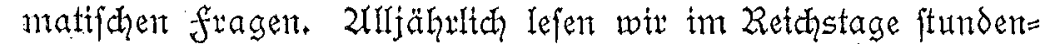
Jange Debatten, ob irgend cine Bumnenfigur Babehofen anbes 
fommen foll oder nidht; ob dies oder jenes $B$ ud verboten werden

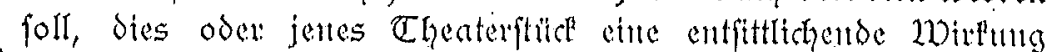
"ausilbl Le. Lhiterdeffen aber geht bas Derderben feinen Bang;

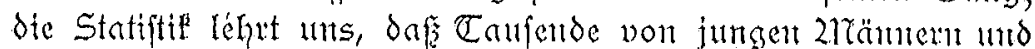
jungen zlidodyen th dent Suntpf des safters verfinfen, fid mit

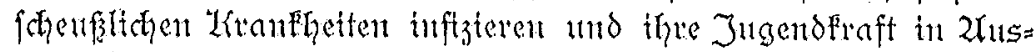
faweifungen vergenden; ftatt htev helfend cunzheifen, werzettelt ntan fetne Bett ntit unerqutalidien Debatten, benutzt diefe entrite frage als Dedrmantel für polttifhe und oogntattache Strettercten. Die franen find die exften gewefen, die de Stthlichettstrage losgelöt haben vont diefen parteifuagen, die die offentliche Utt= fittlidfeit als das betraditet und hingeftellt haben, was fie tit:

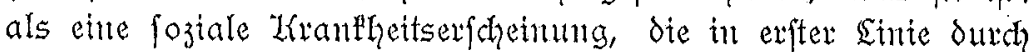
foztale Reformen zut hetren ift. Bebt dem Dolle gefunde 2Doh $=$ turtgen, gebt ifnn billige nnd gefunde Dollsunterhaltungen, gebt ifm Spiel= mo Sportpläze, gebt ifm zitdung und 2luf

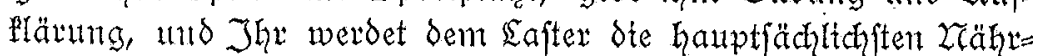
quellen abgrabent.

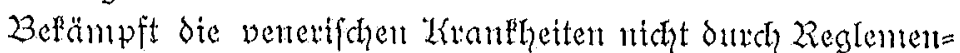
tienting des Safters, fonden bant Krankenhänfer und behandelt die Luantert als Luanpe, ntht als Derbrecher. Dies gilt vor

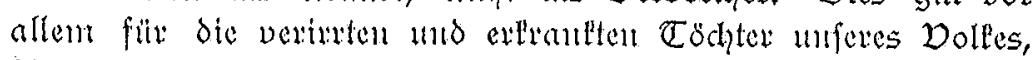
bie man bisher bulfen lief fill: Devbrednen, die all ibnen be= galtgen wurden, ote man mit bem Stempel bev Pontrolltenten Dinte bratomarlte und fie damit ganz in den Sumpf hitabftiefs, ftatt ifuten eine helfende Gand entgegentuftredien.

2luch in dent berilhnten Kantp gegent den Santuts in

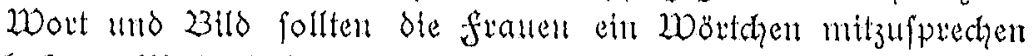
haben, Wohl haben wix franen den Kantpf gegen ben Samuiz in Wont und Bild, dex vonfetten det fonteffonellen Sittlidfetts= vereine aufgenommen wurd, an wht fït fich mit frenden be= grifit, dem naturgentäf ift das weiblidhe Shangefüh befonders

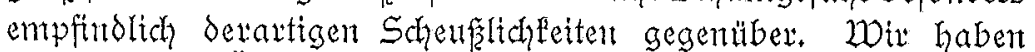

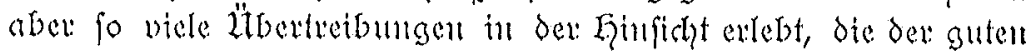

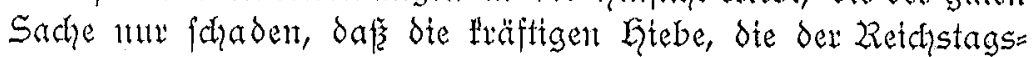
abgeordtete 217üler=217etningen gegen henchelet und ubertrtebene
Priberie geführt hat, gerabe in unferen Kretfen lebhafte Zu=

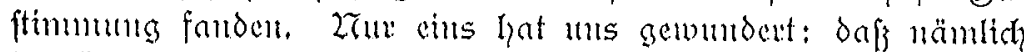
bie fiemen aus betoen Sagen die 2lithilfe ber frauen in diefent Kampfe ablehntent und daf man als bent 27rmbe libe

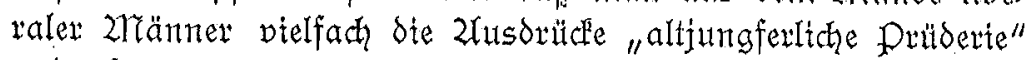

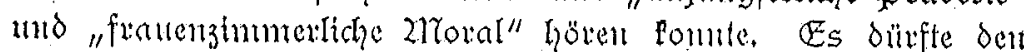
Lewen fdwer fallen, franen namthaft ju madien, die an diefer übertrtebenen pulderte leiden, sie dod mur aus einer vergtf= teten phantafte entfpringt. Jut Brofen und Banzen glaube

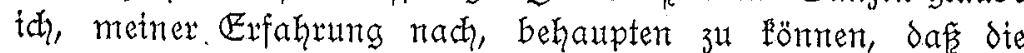
franenwelt fid nod ste unbefangente Reinheit oder reine Utr= befangenheit erfalten hat, unt das Katürlthe nit gefundent Empfinden betradten zu Pönnen; id glaube, wir haben das Unterfhetontgsvermögen zwifhen fenfher Lapthet nub frivo= Ität, zwifhen Equmor und Jote noh nimt verlowen und th

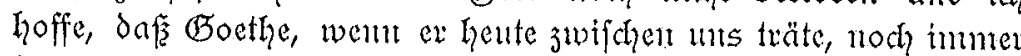
fagen witube: "Willft Du genau erfahren, was fich zient, fo frage nut bet weinen frauen au,"

Wentu uns franen bemod ein groper Teil unferer Gam= lofigfeit verlowen gegangen ift, fo ift das wahthaftig nidht unfere Shuld, fonden die ser zränner, die fie uns geranbt haben. Jdh möhte dies mu dund einige Beifpiele evläutern; Diele Zahre tobte in Beutn der Liampf, of es ben framen geftattet fetu pollte, auf bem Derbed det Ummibuje zu fahten und viele

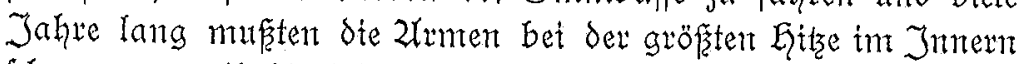
falmoren, wetl die 2hänner für ihre etgente Sittliblett bejorgt waven, wern betnt 2luffteg zum Derded vielletht dew Kundhel etres franenfupes ftatbat wilrde. Ein anderes Betfpiel: Die frende an gefundent Spout, den nicht mut die Sebensluft evigöht, fonden in dem wit aud das befte zrittel zut Gebutg der Sitt=

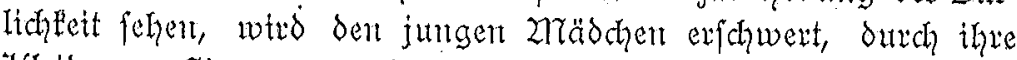

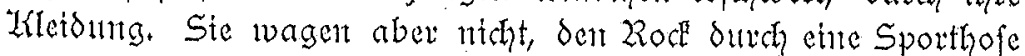

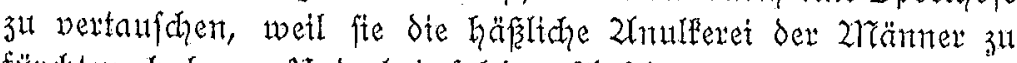

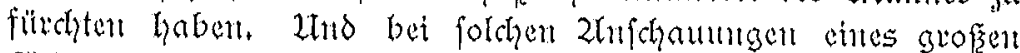
Tetles der 2hännermelt follen ste frauen ifre Gamtofigkett bewafien und nidt pribe werden $p$ ! 


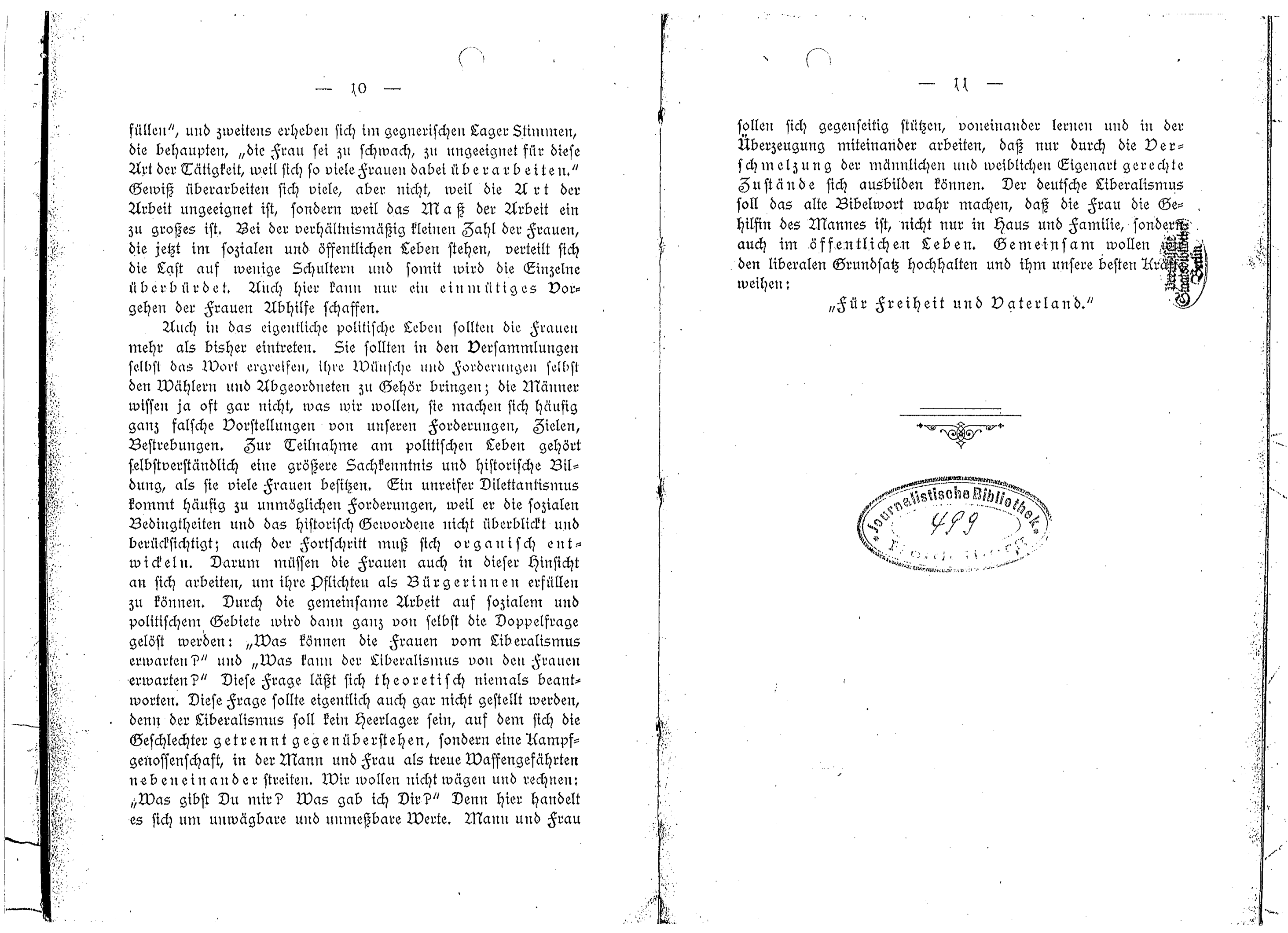

